\title{
A Hybrid Shapeless Radial Basis Function Applied With the Dual Reciprocity Boundary Element Method
}

\author{
KRITTIDEJ CHANTHAWARA \\ Program of Mathematics, Faculty of Science \\ Ubon Ratchathani Rajabhat University \\ Ubon Ratchathani \\ THAILAND \\ Krittidej.c@ubru.ac.th \\ www.ubru.ac.th \\ SAYAN KAENNAKHAM \\ School of Mathematics, Institute of Science \\ Suranaree University of Technology \\ Nakhon Ratchasima \\ THAILAND \\ Sayan_kk@g.sut.ac.th \\ www.sut.ac.th
}

\begin{abstract}
The so-called Dual Reciprocity Boundary Element Method (DRBEM) has been a popular alternative scheme designed to alleviate problems encountered when using the traditional BEM for numerically solving engineering problems that are described by PDEs. The method starts with writing the right-hand-side of Poisson equation as a summation of a pre-chosen multivariate function known as 'Radial Basis Function (RBF)'. Nevertheless, a common undesirable feature of using RBFs is the appearance of the so-called 'shape parameter' whose value greatly affects the solution accuracy. In this work, a new form of RBF containing no shape (so that it can be called 'shapefree/shapeless') is invented, proposed and applied in conjunction with DRBEM is validated numerically. The solutions obtained are compared against both exact ones and those presented in literature where appropriate, for validation. It is found that reasonably and comparatively good approximated solutions of PDEs can still be obtained without the difficulty of choosing a good shape for RBF used.
\end{abstract}

Key-Words: Dual reciprocity, Boundary element method, Shapeless parameter, Radial basis function, Partial differential equation, Numerical solution

Received: November 21, 2020. Revised: March 13, 2021. Accepted: April 1, 2021. Published: May 2, 2021.

\section{Introduction}

It is known that a great amount of engineering and science problems occurring nowadays can be modelled by some forms of mathematical formulas where differential equations (DEs) are amongst those common and popular ones. This inevitably makes finding solutions to these DEs crucial and there are mainly two ways to do this mathematically; analytically and numerically. Unfortunately, most DEs involved are complex and contain several surrounding factors making finding analytic solutions extremely difficult or not possible in many cases. Attempts then have been put into approximating solution using some numerical methods with the use also from the advanced technology in computing systems.

Traditionally, there numerical approaches are well known and have long been used in a wide range of applications and they are finite difference (FD), finite element (FE), and finite volume (FV) method. Appearing as an alternative numerical method over the last two decades, the 
boundary element method (BEM) has become an important tool for solving a wide range of applied sciences and engineering. Like other those traditional methods, BEM is, nevertheless, not without its challenges particularly when applied to nonlinear and/or time-dependence. This remained a pain until 1982 when Nardini and Brebbia [1] proposed a way to overcome the shortcoming. The approach involves splitting the solution into two parts; complementary solutions of its homogeneous form and the particular solutions of the inhomogeneous counterpart, and they named it as 'Dual Reciprocity Boundary Element Method (DRBEM). Since the particular solutions are not always available especially in complex problems, the inhomogeneous term of the PDE is approximated by a series of simple functions and transformed to the boundary integrals employing particular solutions of considered problem. The most widely used approximating functions in DRBEM are radial basis functions (RBFs) for which particular solutions can be easily determined [2].

Radial Basis Functions (RBF), $\varphi$, are commonly found as multivariate functions whose values are dependent only on the distance from the origin. This means that $\varphi(\mathbf{x})=\varphi(r) \in \square$ with $\mathbf{x} \in \square^{n} \quad$ and $r \in \square$; or, in other words, on the distance from a point of a given set $\left\{\mathbf{x}_{j}\right\}$, and $\varphi\left(\mathbf{x}-\mathbf{x}_{j}\right)=\varphi\left(r_{j}\right) \in \square$. Here, $r_{j}=\left\|\mathbf{x}-\mathbf{x}_{j}\right\|_{2}$ is the Euclidean distance and any function $\varphi$ satisfying $\varphi(\mathbf{x})=\varphi\left(\|\mathbf{x}\|_{2}\right)$ is a radial function and have been receiving a great amount of interest from researchers from many branches of applications [3]. Some of the most popular ones are [1];

- Gaussian (GS) : $\varphi(r)=\exp ^{-(\varepsilon r)^{2}}$

- Inverse Multiquadric (IMQ) : $\varphi(r)=1 / \sqrt{1+(\varepsilon r)^{2}}$

- Multiquadric (MQ) : $\varphi(r)=\sqrt{1+(\varepsilon r)^{2}}$
- Inverse quadratic (IQ) : $\varphi(r)=1 /\left[1+(\varepsilon r)^{2}\right]$

- Wendland (WL) :

$$
\varphi(r)=(1-\varepsilon r)^{8}\left(32(\varepsilon r)^{3}+25(\varepsilon r)^{2}+8 \varepsilon r+1\right)
$$

- Cubic Matérn (CU) :

$$
\varphi(r)=\left(15+15 \varepsilon r+6(\varepsilon r)^{2}+(\varepsilon r)^{3}\right)
$$

Here, $\varepsilon$ is called shape parameter, very often determined by the user.

As can be seen from above list, one of the most challenging tasks for utilizing RBFs, finding a suitable or optimal shape parameter, $\varepsilon$, is crucial and this tasks is not straightforward. In the past, some attempts to pinpoint the optimal value of $\varepsilon$ involve the classic work of Hardy [4] where it was shown that by fixing the shape at $\varepsilon=1 /(0.815 d)$, where $d=(1 / N) \sum_{i=1}^{N} d_{i}$, and $d_{i}$ is the distance from the node to its nearest neighbour, good results should be anticipated. Also, in the work of Franke and Schaback [5] where the choice of a fixed shape of the form $\varepsilon=0.8 \sqrt{N} / D$ with $D$ being the diameter of the smallest circle containing all data nodes, can also be a good alternative. In 2000, Zhang et al. [6] demonstrated and concluded that the optimal shape parameter is highly problem dependent. In 2002, Wang and Lui [7] pointed out that by analysing the condition number of the collocation matrix, a suitable range of derivable values of $\varepsilon$ can be found. Later in 2003, Lee et al. [8] suggested that the final numerical solutions obtained are found to be less affected by the method when the approximation is applied locally rather than globally. A rather recent work is the selection of an interval for variable shape parameter by Biazar and Hosami in 2016 [9], where a novel algorithm for determining and interval was proposed, please see also [10-13] and references herein. Another confirmation on problem-dependent nature of shape value was confirmed in the work of Davydov and Oanh [14]. A more recent work is the application of the traditional Kansa collocation to linear PDE based on a variable shape parameter called symmetric 
variable shape parameter (SVSP) carried out by Ranjbar [15]. Some relevant studies can be found in [16] and [17].

Additionally, there have also been some researches carried out on finding the optimal shape automatically based on some computational factors already involved in the system. Some works regarding this figure are listed in Table 1.

Table 1. Some choices proposed in literature

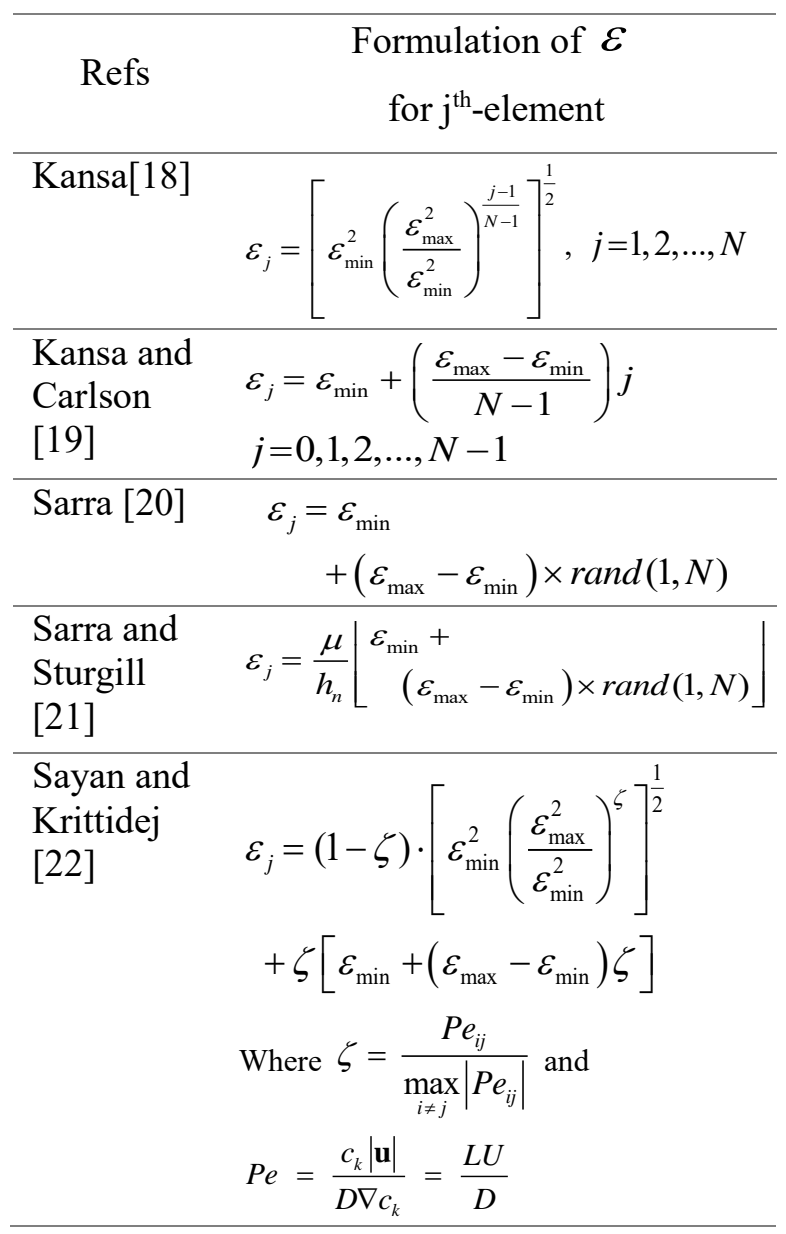

Up to this point, it has to be noted that radial basis functions were originally invented for applying to function approximation and interpolation, and later applied in the context of meshfree/meshless numerical methods for solving PDEs. It means that the issues related to the shape parameter is based on such problems. Investigations mentioned so far, also some in Table 1, are not yet related to DRBEM (except only one done in [22]). This prompts an inspiration of this work.

Despite all the attempts being put into this topic of optimal shape finding procedure, it remains open topic and is still under a great intention of nowadays research. In this work, a new form of RBF containing no 'ad-hoc' shape parameters is proposed and its performance is investigated in the context of DRBEM. Section II provides the fundamental concept of the DRBEM where Section III contains the information about the proposed RBF. Some numerical testing cases are presented in Section IV and some important findings and conclusions are drawn in Section V.

\section{The Dual Reciprocity Boundary Element Method (DRBEM)}

The mathematical construction of the dual reciprocity boundary element method (DRBEM) can start with the Poisson equation as follows;

$$
\nabla^{2} u=b(x, y)
$$

Where the solution to the above problem can be expressed as the sum of the solution of homogenous and a particular solution as;

$$
u=u_{h}+\hat{u}
$$

Where $u_{h}$ is the solution of the homogeneous equation and $\hat{u}$ is a particular solution.

If there are $\mathrm{N}$ boundary nodes and $\mathrm{L}$ internal nodes, there will be $\mathrm{N}+\mathrm{L}$ interpolation functions, $f_{j}$ and consequently $\mathrm{N}+\mathrm{L}$ particular solutions, $\hat{u}_{j}$. The approximation of $b$ over domain $\Omega$ is written, for an $i^{\text {th }}-$ node, in the following form.

$$
b_{i}(x, y) \approx \sum_{j=1}^{N+L} \alpha_{j} f_{i j}(x, y)
$$

Then we have a matrix equation for the unknown coefficients $\alpha_{j}: j=1,2,3, \ldots, N+L$ as follows;

$$
\mathbf{b}=\mathbf{F} \alpha
$$

With the following detains. 
$\mathbf{b}=\left[\begin{array}{c}b_{1}(x, y) \\ b_{2}(x, y) \\ \vdots \\ b_{N+L}(x, y)\end{array}\right], \alpha=\left[\begin{array}{c}\alpha_{1} \\ \alpha_{2} \\ \vdots \\ \alpha_{N+L}\end{array}\right]$, and

$\mathbf{F}=\left[\begin{array}{cccc}f_{11}(x, y) & f_{12}(x, y) & \cdots & f_{1(\mathrm{~N}+\mathrm{L})}(x, y) \\ f_{21}(x, y) & f_{22}(x, y) & \cdots & f_{2(\mathrm{~N}+\mathrm{L})}(x, y) \\ \vdots & \vdots & \ddots & \vdots \\ f_{(\mathrm{N}+\mathrm{L}) 1}(x, y) & f_{(\mathrm{N}+\mathrm{L}) 2}(x, y) & \cdots & f_{(\mathrm{N}+\mathrm{L})(\mathrm{N}+\mathrm{L})}(x, y)\end{array}\right]$

The particular solution, $\hat{u}_{j}$, and the interpolation function, $f_{j}$, are linked through the relation.

$$
\nabla^{2} \hat{u}_{j}=f_{j}
$$

Substituting Eq. (5) into Eq. (3) leads;

$$
b=\sum_{j=1}^{N+L} \alpha\left(\nabla^{2} \hat{u}_{j}\right)
$$

Therefore, from Eq. (1) and with the expression of $b$ in Eq. (3), we then obtain its equivalent integral form expressed as;

$$
\begin{aligned}
& c_{i} u_{i}+\int_{\Gamma} q^{*} u d \Gamma-\int_{\Gamma} u^{*} q d \Gamma \\
& =\sum_{j=1}^{N+L} \alpha_{j}\left(c_{i} \hat{u}_{i j}+\int_{\Gamma} q^{*} \hat{u}_{j} d \Gamma-\int_{\Gamma} u^{*} \hat{q}_{j} d \Gamma\right)
\end{aligned}
$$

Where $u^{*}$ is the fundamental solution and the term $\hat{q}_{j}$ is defined as $\hat{q}_{j}=\frac{\partial \hat{u}_{j}}{\partial \mathbf{n}}$, where $\mathbf{n}$ is the unit outward normal to $\Gamma_{\Gamma}$, and can be written as follows;

$$
\hat{q}_{j}=\frac{\partial \hat{u}_{j}}{\partial x} \frac{\partial x}{\partial n}+\frac{\partial \hat{u}_{j}}{\partial y} \frac{\partial y}{\partial n}
$$

Applying Green's theorem, the boundary element approximation to Eq. (7), then it becomes, at an $\mathrm{i}^{\text {th }}$-node;

$$
\begin{aligned}
c_{i} u_{i} & +\sum_{k=1}^{N} \int_{\Gamma_{k}} q^{*} u d \Gamma-\sum_{k=1}^{N} \int_{\Gamma_{k}} u^{*} q d \Gamma \\
& =\sum_{j=1}^{N+L} \alpha_{j}\left(c_{i} \hat{u}_{i j}+\sum_{k=1}^{N} \int_{\Gamma_{k}} q^{*} \hat{u}_{j} d \Gamma-\sum_{k=1}^{N} \int_{\Gamma_{k}} u^{*} \hat{q}_{j} d \Gamma\right)
\end{aligned}
$$

For $i=1, \ldots, \mathrm{N}$.

After introducing the interpolation function and integrating over each boundary elements, Eq. (9) can be re-written in terms of nodal values as;

$$
\begin{aligned}
c_{i} u_{i} & +\sum_{k=1}^{N} H_{i k} u_{k}-\sum_{k=1}^{N} G_{i k} q_{k} \\
& =\sum_{j=1}^{N+L} \alpha_{j}\left(c_{i} \hat{u}_{i j}+\sum_{k=1}^{N} H_{i k} \hat{u}_{k j}-\sum_{k=1}^{N} G_{i k} \hat{q}_{k j}\right)
\end{aligned}
$$

Where the definition of the terms $H_{i k}$ and $G_{i k}$ can be found in Chanthawara et.al. [2] and Toutip [23]. The index $k$ is used for the boundary nodes which are the field points. After application to all boundary nodes, using a collocation technique, Eq. (10) can be compactly expressed in matrix form as follows;

$$
\mathbf{H u}-\mathbf{G q}=(\mathbf{H} \hat{\mathbf{U}}-\mathbf{G} \hat{\mathbf{Q}}) \alpha
$$

By substituting $\alpha=\mathbf{F}^{-1} \mathbf{b}$ from Eq. (4), into Eq. (11) making the right hand side of Eq. (11) a known vector. Therefore, it can be rewritten as;

$$
\mathbf{H u}-\mathbf{G q}=\mathbf{d}
$$

where $\quad \mathbf{d}=(\mathbf{H} \hat{\mathbf{U}}-\mathbf{G} \hat{\mathbf{Q}}) \mathbf{F}^{-1} \mathbf{b} . \quad$ Applying boundary condition(s) to Eq. (12), then it can be seen as the simple form as follow;

$$
\mathbf{A x}=\mathbf{y}
$$

Where $\mathbf{x}$ contains $N$ unknown boundary values of $u$ 's and q's .

After Eq. (13) is solved using standard techniques such as Gaussian elimination, the values at any internal node can be calculated, i.e. $c_{i}=1$ as expressed in Eq. (10) where each one involves a separate multiplication of known vectors and matrices.

$$
\begin{aligned}
u_{i} & =-\sum_{k=1}^{N} H_{i k} u_{k}+\sum_{k=1}^{N} G_{i k} q_{k} \\
& =\sum_{j=1}^{N+L} \alpha_{j}\left(c_{i} \hat{u}_{i j}+\sum_{k=1}^{N} H_{i k} \hat{u}_{k j}-\sum_{k=1}^{N} G_{i k} \hat{q}_{k j}\right)
\end{aligned}
$$

The further use of the DRBEM derivation explained so far depends on the form of the 
governing equation under investigation. Therefore, examples where DRBEM is being implemented for each case and type of equations are later provided in Section 4.

\section{The Proposed Hybrid RBF}

The idea of combining different forms of RBF is becoming an alternative choice. A recent study on this matter was done in 2018 where a combination of Gaussian and Cubic forms of RBF is formed by Mishra et.al. [24]. The main application of their work is on interpolation of data and it is to be noted that the attempt to find an adequate choice of $\varepsilon$ still remains a burden, requiring extra treatments. In this work, two of the most popular forms of RBF defined as follows are under investigation;

1. Multiquadric (MQ) :

$$
\varphi^{M Q}\left(\left\|\mathbf{x}-\mathbf{x}_{j}\right\|_{2}\right)=\left[\varepsilon^{2}+\left(\left\|\mathbf{x}-\mathbf{x}_{j}\right\|_{2}\right)^{2}\right]^{\frac{1}{2}}
$$

2. Polyharmonic (PHC) :

$$
\varphi^{P H C}\left(\left\|\mathbf{x}-\mathbf{x}_{j}\right\|\right)=\left(\left\|\mathbf{x}-\mathbf{x}_{j}\right\|_{2}\right)^{5}
$$

In this work, the shape parameter $\varepsilon$ is now to be fixed at 1 , and a new locally self-autoadaptive weight $\omega$ is proposed, as follows;

$$
\begin{gathered}
\varphi_{i j}^{H y B}\left(\left\|\mathbf{x}_{i}-\mathbf{x}_{j}\right\|_{2}\right) \\
=\left(1-\omega_{i j}\right)\left[1+\left(\left\|\mathbf{x}-\mathbf{x}_{j}\right\|_{2}\right)^{2}\right]^{\frac{1}{2}} \\
+\omega_{i j}\left(\left\|\mathbf{x}_{i}-\mathbf{x}_{j}\right\|_{2}\right)^{5}
\end{gathered}
$$

where $\omega_{i j}=\left\|\mathbf{x}_{i}-\mathbf{x}_{j}\right\|_{2} /\left[\max _{1 \leq i, j \leq N^{x}}\left\|\mathbf{x}_{i}-\mathbf{x}_{j}\right\|_{2}\right] \quad$ is adaptable purely based on the location of the interpolation vector $\mathbf{x}_{j}$ and the centre at hand, $\mathbf{x}_{i}$. This shall act as a self-adaptive parameter with no further input from user. This proposed RBF shall be referred to as 'HyB' for short throughout the document.

\section{Numerical Experiments and General Discussion}

All numerical simulations under this investigation were carried out on an $\operatorname{Intel}(\mathrm{R})$ Core(TM) i7-5500U CPU @ 2.4GHz with 8.00 GB of RAM. It has to be mentioned also that for comparison purpose, all results produced by $\mathrm{MQ}$, PHC and HyB are shown against one another in all the cases. For MQ, in particular, the best choice of shape parameter for each experiment was obtained purely by carrying out a large number of simulations and employing the error norm related and they are listed in the following Section.

\subsection{Error norms}

All numerical solutions obtained from the whole experiment are validated mainly by comparing to the exact or analytical solutions. For this, the following error norms are used;

(1) Maximum Error $\left(L_{\infty}\right)$;

$$
L_{\infty}=\max _{1 \leq i \leq N^{\chi}}\left|u^{e x t}\left(\mathbf{x}_{i}\right)-u^{a p p x .}\left(\mathbf{x}_{i}\right)\right|
$$

(2) Root-Mean-Square Error $\left(L_{r m s}\right)$;

$L_{r m s}=\left(\frac{1}{N^{\chi}} \sum_{j=1}^{N^{\chi}}\left(u^{e x t}\left(\mathbf{x}_{i}\right)-u^{a p p x .}\left(\mathbf{x}_{i}\right)\right)^{2}\right)^{1 / 2}$

(3) Percentage Relative Error ( $\%-$ Rel.Err. $\left.\left(\mathbf{x}_{i}\right)\right)$

$$
\begin{aligned}
& \%-\text { Rel.Err. }\left(\mathbf{x}_{i}\right) \\
& \qquad=\frac{\left|u^{\text {ext }}\left(\mathbf{x}_{i}\right)-u^{\text {appx. }}\left(\mathbf{x}_{i}\right)\right|}{\left|u^{\text {ext }}\left(\mathbf{x}_{i}\right)\right|} \times 100
\end{aligned}
$$

(4) Absolute Error ( Abs.Err.)

$$
\operatorname{Abs.Err.}\left(\mathbf{x}_{j}\right)=\left|u^{\text {ext }}\left(\mathbf{x}_{i}\right)-u^{a p p x .}\left(\mathbf{x}_{i}\right)\right|
$$

Where $u^{e x t}\left(\mathbf{x}_{i}\right), u^{a p p x .}\left(\mathbf{x}_{i}\right)$ are the approximate and exact solution respectively, computed at node $\mathbf{x}_{i}$ with $N^{x}$ being the total number of nodes.

\subsection{Test case 1: interpolation problem}


The interpolation problem starts with a set of discrete data $\mathbf{X}=\left\{\mathbf{x}_{i}\right\}_{i=1}^{N}, \mathbf{x}_{i} \in \square^{\mathrm{d}}$ where for each $\mathbf{x}_{i}$ there is its corresponding real value $y_{i} \in \square$, then the task is to construct a continuous function $\wp(\mathrm{x}): \square^{\mathrm{d}} \rightarrow \square$ such that;

$$
\wp\left(\mathbf{x}_{i}\right)=y_{i}, i=1,2, \ldots, N
$$

Since the main concept of DRBEM is to approximate term $b_{i}(x, y)$, in Eq. (3), by a linear combination of RBF as a collocation manner, the first example to test out the proposed RBF here is taken to be a benchmark and well-known Franke-type function [2]. The function defined on a unit square-domain is expressed as;

$$
\begin{gathered}
f(x, y)=0.75 \exp \left[-\frac{(9 x-2)^{2}}{4}-\frac{(9 y-2)^{2}}{4}\right] \\
+0.75 \exp \left[-\frac{(9 x+1)^{2}}{49}-\frac{(9 y+1)}{10}\right] \\
+0.5 \exp \left[-\frac{(9 x-7)^{2}}{4}-\frac{(9 y-3)^{2}}{4}\right] \\
-0.2 \exp \left[-(9 x-4)^{2}-(9 y-7)^{2}\right]
\end{gathered}
$$

The interpolation function, $\tilde{f}\left(\mathbf{x}_{i}\right)$ at the $i-t h$ centre node, $\mathbf{x}_{i}$, is defined as a linear combination of the inverse quadratic radial basis function as;

$$
\tilde{f}\left(\mathbf{x}_{i}\right)=\sum_{j=1}^{N^{\chi}} \alpha_{j} \varphi_{i j}^{H y B}\left(\left\|\mathbf{x}_{i}-\mathbf{x}_{j}\right\|_{2}\right)
$$

With $\tilde{f}\left(\mathbf{x}_{i}\right)=f\left(\mathbf{x}_{i}\right)$ for all $i=1,2, \ldots, N^{\chi}$ with a set of $N^{\chi}=N+L$ computational nodes uniformly-distributed over the unit square (i.e.

\begin{tabular}{|c|c|c|c|c|c|c|}
\hline \multirow{2}{*}{$\mathbf{x}_{j}=(x, y)$} & \multicolumn{3}{|c|}{ Abs.Err. $\left(\mathbf{x}_{j}\right)$ With $N^{\chi}=4$} & \multicolumn{3}{|c|}{ Abs.Err. $\left(\mathbf{x}_{j}\right)$ With $N^{\chi}=225$} \\
\hline & MQ & PHC & HyB & MQ & PHC & HyB \\
\hline
\end{tabular}
the summation of internal and boundary nodes). By imposing this function on all nodes, it leads to the linear system expressed as;

Table 2. A comparison of absolute errors measured at a set of selected locations when using two levels of node density; $N^{x}=49$ and $N^{x}=225$.
Where

$$
\mathbf{A}=\left[\begin{array}{cccc}
\varphi^{H y B}\left(r_{11}\right) & \varphi^{H y B}\left(r_{12}\right) & \ldots & \varphi^{H y B}\left(r_{1 N^{\chi}}\right) \\
\varphi^{H y B}\left(r_{21}\right) & \varphi^{H y B}\left(r_{22}\right) & \ldots & \varphi^{H y B}\left(r_{2 N^{\chi}}\right) \\
\vdots & \vdots & \ddots & \vdots \\
\varphi^{H y B}\left(r_{N^{x_{1}}}\right) & \varphi^{H y B}\left(r_{N^{x_{2}}}\right) & \ldots & \varphi^{H y B}\left(r_{N^{z} N^{x}}\right)
\end{array}\right]_{N^{x} \times N^{x}}
$$

Where $r_{i j}=\left\|\mathbf{x}_{i}-\mathbf{x}_{j}\right\|_{2}$. The coefficient matrix; $\boldsymbol{\alpha}=\left[\begin{array}{llll}\alpha_{1} & \alpha_{2} & \ldots & \alpha_{N^{x}}\end{array}\right]^{\mathrm{T}}$, and with the known vector function $\mathbf{F}=\left[\begin{array}{llll}\tilde{f}\left(\mathbf{x}_{1}\right) & \tilde{f}\left(\mathbf{x}_{2}\right) & \ldots & \tilde{f}\left(\mathbf{x}_{N^{x}}\right)\end{array}\right]^{T} . \quad$ By the well-known Gauss-Seidel method, the coefficient matrix $\boldsymbol{\alpha}$ is easily obtained and it will then be used to interpolate the value of $f(x, y)$ at a new set of interpolation nodes via. the same linear summation as defined above.

Table 2 lists the measurement on absolute errors Abs.Err. $\left(\mathbf{x}_{j}\right)$ taken at some selected locations over the domain with two cases; $N^{\chi}=49$ and $N^{\chi}=225$. It can be clearly seen from the Table that the increase in the number of computational nodes, a better quality of results can be expected from all three RBFs. It should be noted, nevertheless, that where MQ and HyB remain nearly the same level of solution quality, it is $\mathrm{PHC}$ that has the biggest absolute errors in overall. Also, where MQ requires a great amount of effort for finding the best shape for each case, this burden has no effect on HyB indicating a much more convenient practical use. For $N^{\chi}=49$, the computed solution obtained from HyB is plotted against the exact ones and is illustrated in Fig. 1. 


\begin{tabular}{ccccccc}
\hline & $\left(\varepsilon_{\text {best }}=4.5\right)$ & \multicolumn{5}{c}{$\left(\varepsilon_{\text {best }}=0.74\right)$} \\
\hline$(0.00,0.66)$ & $1.70 \mathrm{E}-03$ & $3.37 \mathrm{E}-01$ & $2.51 \mathrm{E}-03$ & $0.25 \mathrm{E}-04$ & $1.02 \mathrm{E}-03$ & $0.21 \mathrm{E}-04$ \\
$(0.00,1.00)$ & $1.64 \mathrm{E}-02$ & $2.21 \mathrm{E}-01$ & $5.23 \mathrm{E}-03$ & $2.33 \mathrm{E}-04$ & $0.27 \mathrm{E}-03$ & $1.25 \mathrm{E}-04$ \\
$(0.33,0.00)$ & $9.99 \mathrm{E}-02$ & $4.09 \mathrm{E}-01$ & $8.15 \mathrm{E}-02$ & $5.26 \mathrm{E}-03$ & $2.66 \mathrm{E}-02$ & $5.66 \mathrm{E}-04$ \\
$(0.33,0.33)$ & $9.99 \mathrm{E}-03$ & $3.22 \mathrm{E}-02$ & $0.25 \mathrm{E}-04$ & $1.02 \mathrm{E}-04$ & $9.21 \mathrm{E}-02$ & $0.27 \mathrm{E}-05$ \\
$(0.33,0.66)$ & $6.29 \mathrm{E}-02$ & $2.66 \mathrm{E}-01$ & $1.22 \mathrm{E}-04$ & $2.39 \mathrm{E}-04$ & $8.05 \mathrm{E}-03$ & $5.11 \mathrm{E}-04$ \\
$(0.33,1.00)$ & $9.20 \mathrm{E}-02$ & $2.09 \mathrm{E}-02$ & $5.04 \mathrm{E}-03$ & $0.15 \mathrm{E}-03$ & $9.20 \mathrm{E}-03$ & $0.24 \mathrm{E}-05$ \\
\hline
\end{tabular}

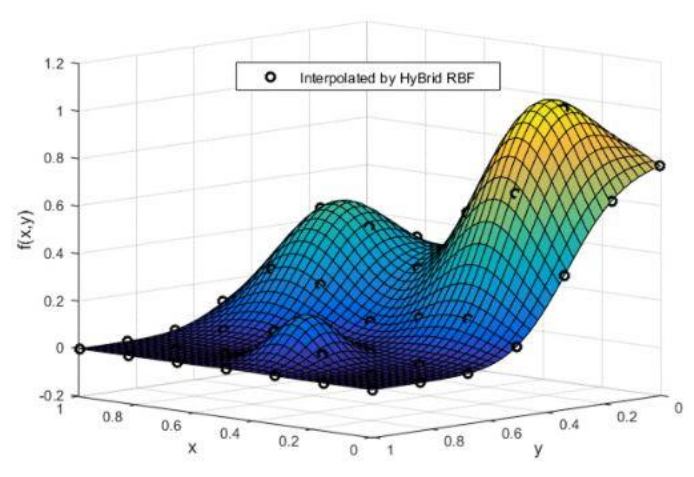

Fig. 1. Exact solution profile plotted against those interpolated by the proposed RBF using $N^{\chi}=49$.

\subsection{Test case 2: Poisson with nonrectangular domain}

The second case used to monitor the quality of results produced by the proposed RBF is a Possion form defined over a nonrectangular domain. This problem has recently been studied numerically by Kaennakham and Chuathong [25] where a global collocation meshfree scheme was applied.

The equation is shown below;

$$
\frac{\partial^{2} u}{\partial x^{2}}+\frac{\partial^{2} u}{\partial y^{2}}=-x^{2}
$$

This is defined on the domain with an elliptical boundary, expressed as;

$$
\frac{x^{2}}{4}+y^{2}=1
$$

Where the boundary condition is taken directly from the exact solution which is expressed as follows;

$$
u(x, y)=-\frac{1}{246}\left(50 x^{2}-8 y^{2}+33.6\right) \vartheta
$$

Where

$$
\vartheta=\left(\frac{x^{2}}{4}+y^{2}-1\right)
$$

The DRBEM implementation explained in Section 2 is now straightforwardly done by rearranging Eq (12);

$$
\mathbf{H u}=\mathbf{G q}+\mathbf{d}
$$

Since $\mathbf{d}$ is a known vector and after applying the boundary conditions, it then can be seen in the form of Eq . (13).

In this example, the main focus is paid to the effect of the density of nodes contained in the domain and for this, at least 4 levels of node density are investigated. Table 3 displays four levels of density where the number of boundary nodes is kept constant at 40 for all cases. With two error norms, $L_{r m s}$ and $L_{\infty}$, under consideration, it is found once more time that MQ and $\mathrm{HyB}$ can produce high quality of accuracy whereas PHC has the lowest accuracy for node densities. The main shortcoming, however, is still with the use of MQ where the best shape, $\varepsilon_{\text {Best }}$, is found not to reveal any correlation with the increase of nodes confirming the problemdependent of MQ. Without the extra task of finding an optimal shape, HyB, on the other hand, is seen to gradually produce better results when nodes are denser (with $L_{r m s}=5.41 E-04$ for $N^{\chi}=91$ to $L_{r m s}=0.25 E-04$ for $N^{\chi}=431$ ). Fig. 2 depicts the problem domain and solutions 
produced by HyB plotted against the exact solutions.

Table 3. Root mean square $\left(L_{r m s}\right)$ and maximum error norms $\left(L_{\infty}\right)$ measured from using different numbers of computational nodes.

\begin{tabular}{lrrrrrrrrr}
\hline & \multicolumn{2}{c}{$N^{\chi}=91$} & \multicolumn{2}{c}{$N^{\chi}=165$} & \multicolumn{2}{c}{$N^{\chi}=217$} & \multicolumn{2}{c}{$N^{\chi}=431$} \\
\cline { 2 - 9 } & \multicolumn{1}{c}{$L_{r m s}$} & \multicolumn{1}{c}{$L_{\infty}$} & \multicolumn{1}{c}{$L_{r m s}$} & \multicolumn{1}{c}{$L_{\infty}$} & \multicolumn{1}{c}{$L_{r m s}$} & \multicolumn{1}{c}{$L_{\infty}$} & \multicolumn{1}{c}{$L_{r m s}$} & \multicolumn{1}{c}{$L_{\infty}$} \\
\hline MQ & $2.25 \mathrm{E}-04$ & $2.01 \mathrm{E}-03$ & $1.89 \mathrm{E}-03$ & $2.08 \mathrm{E}-02$ & $2.14 \mathrm{E}-03$ & $6.25 \mathrm{E}-02$ & $8.06 \mathrm{E}-04$ & $6.26 \mathrm{E}-03$ \\
$\left(\varepsilon_{\text {Best }}=\right)$ & $(11.25)$ & $(11.25)$ & $(4.05)$ & $(4.05)$ & $(1.58)$ & $(1.58)$ & $(21.33)$ & $(21.33)$ \\
\hline PHC & $1.96 \mathrm{E}-03$ & $2.11 \mathrm{E}-02$ & $1.58 \mathrm{E}-03$ & $3.45 \mathrm{E}-02$ & $2.03 \mathrm{E}-03$ & $6.01 \mathrm{E}-02$ & $7.12 \mathrm{E}-03$ & $3.25 \mathrm{E}-02$ \\
\hline HyB & $5.41 \mathrm{E}-04$ & $1.77 \mathrm{E}-03$ & $3.02 \mathrm{E}-04$ & $8.02 \mathrm{E}-03$ & $1.21 \mathrm{E}-04$ & $0.59 \mathrm{E}-04$ & $0.25 \mathrm{E}-04$ & $2.11 \mathrm{E}-04$ \\
\hline
\end{tabular}
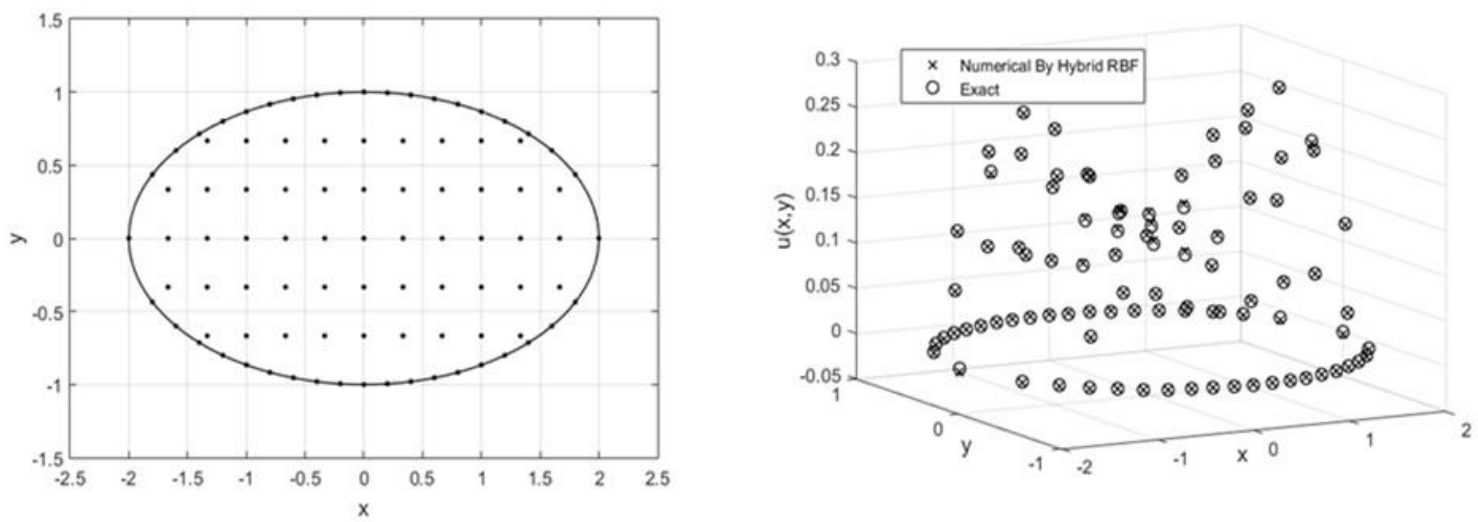

Fig. 2. Above) Problem domain with $N^{\chi}=91$ computational nodes, and below) solutions comparison between those produced by the HyB and the exact ones.

\subsection{Test case 3: transient convection- diffusion problem}

Convection diffusion problems are known to be governed PDE mathematical models and they are found to appear in many branches of sciences and engineering such as biological, physical chemical, physical in fluid mechanics, astrophysics, meteorology, and multiphase flow in oil reservoirs, polymer flow and many other areas [26].

The unsteady-state form of the equation can be represented by;

$$
\begin{aligned}
\frac{\partial u}{\partial t} & +V_{x} \frac{\partial u}{\partial x}+V_{y} \frac{\partial u}{\partial y} \\
& =\eta_{x} \frac{\partial^{2} u}{\partial x^{2}}+\eta_{y} \frac{\partial^{2} u}{\partial y^{2}}-\beta u+g(x, y, t)
\end{aligned}
$$

Where $V_{x}, V_{y}$ are convection coefficients, and $\eta_{x}, \eta_{y}$ are diffusion coefficients. The last two terms $\beta u$ and the source term $g(x, y, t)$ are additional and needed only in specific cases. For the test cases studied in this work, it is set that $\eta_{x}=\eta_{y}=\eta$ and then it leads to the following expression; 


$$
\begin{gathered}
\frac{\partial u}{\partial t}+V_{x} \frac{\partial u}{\partial x}+V_{y} \frac{\partial u}{\partial y}+\beta u-g(x) \\
=\eta\left(\frac{\partial^{2} u}{\partial x^{2}}+\frac{\partial^{2} u}{\partial y^{2}}\right)
\end{gathered}
$$

Leading to;

$\frac{\partial^{2} u}{\partial x^{2}}+\frac{\partial^{2} u}{\partial y^{2}}$

$=\frac{1}{\eta}\left(\frac{\partial u}{\partial t}+\left(V_{x} \frac{\partial u}{\partial x}+V_{y} \frac{\partial u}{\partial y}\right)+\beta u-g(x)\right)$

Subject to the initial condition $u(x, y, 0)=\psi_{1}(x, y)$ and the boundary condition $u(x, y, t)=\psi_{2}(x, y, t)$ with $t>0$ and $\Omega$ is a domain of the problem, $\partial \Omega$ is its boundary, $\psi_{1}, \psi_{2}$ are known functions.

In this case, we set $V_{x}=V_{y}=0.8$ and $\eta_{x}=\eta_{y}=0.01_{\text {with }}$ zero sink and zero source terms, i.e. $\beta u=g(x)=0$. The governing equation is of the form as shown below;

$$
\frac{\partial^{2} u}{\partial x^{2}}+\frac{\partial^{2} u}{\partial y^{2}}=\frac{1}{0.01}\left(\frac{\partial u}{\partial t}+0.8\left(\frac{\partial u}{\partial x}+\frac{\partial u}{\partial y}\right)\right)
$$

Where

$(x, y) \in D=\left\{(x, y) \in \square^{2} \mid 0.5<x, y<2\right\}, t \in(0, T]$

. Its exact solution is defined as follows;

$$
u(x, y, t)=\frac{1}{4 t+1} \exp (\xi),
$$

Where

$$
\xi(x, y, t)=-\frac{(x-0.8 t-0.5)^{2}}{0.01(4 t+1)}-\frac{(y-0.8 t-0.5)^{2}}{0.01(4 t+1)}
$$

The implementation of DRBEM to the type of problem starts with approximating Eq. (1) by;

$$
b=\frac{1}{0.01}\left(\frac{\partial u}{\partial t}+\left(0.8 \frac{\partial u}{\partial x}+0.8 \frac{\partial u}{\partial y}\right)\right)
$$

For the time derivative, the forward difference method is expressed as $\dot{u}=\frac{\partial u}{\partial t}=\frac{u^{t+1}-u^{t}}{\Delta t}$;
Substituting $\frac{\partial u}{\partial x}=\frac{\partial \mathrm{F}}{\partial x} \mathbf{F}^{-1} \mathbf{u}, \frac{\partial u}{\partial y}=\frac{\partial \mathrm{F}}{\partial y} \mathbf{F}^{-1} \mathbf{u} \quad$ and Eq. (33) into Eq. (31) and by setting.

$$
\mathbf{C}=0.8 \frac{\partial \mathrm{F}}{\partial x} \mathbf{F}^{-1}+0.8 \frac{\partial \mathrm{F}}{\partial y} \mathbf{F}^{-1}
$$

The following expressions is obtained.

$$
\mathbf{H u}-\mathbf{G q}=\frac{1}{0.01} \mathbf{S}(\dot{u}+\mathbf{C u})
$$

Let $\mathbf{R}=\frac{1}{0.01} \mathbf{S}$, it leads to;

$$
\mathbf{H u}-\mathbf{G q}=\mathbf{R}(\dot{u}+\mathbf{C u})
$$

And then rearranging the time-derivative term with the forward difference, it becomes;

$$
\left(\frac{\mathbf{R}}{\Delta \mathbf{t}}+\mathbf{R C}-\mathbf{H}\right) \mathbf{u}^{\mathbf{t}+1}+\mathbf{G} \mathbf{q}^{\mathbf{t}+1}=\frac{\mathbf{R}}{\Delta \mathbf{t}} \mathbf{u}^{\mathbf{t}}
$$

Note that the elements of matrices $\mathbf{H}, \mathbf{G}, \mathbf{R}$ depend only on geometrical data. Thus, they can all be computed ones and stored.

Table 4 presents the percentage relative error measured at 7 selected points over the problem domain at two time levels ; $t=0.5$ and $t=1.0$. These two sets of solutions use the same computational setups; $N^{x}=91$ and $\Delta t=0.005$. At early state of time, i.e. $t=0.5$, it is observed that all three RBF types provide approximately the same level of accuracy. When time progresses, it is interestingly seen that both MQ and PHC are found to have lost their capability of producing better accuracy. It is actually solutions produced by HyB that are seen to remain in good quality even when time increases. This indicates another desirable aspect of the newly proposed form of RBF.

With the maximum error norm $\left(L_{\infty}\right)$ used to monitor the growth in error when increasing time further, i.e. $1.1 \leq t \leq 2.0$ with $N^{x}=144$, moreover, Fig. 3 reveals that HyB is considerably less sensitive to the number of time increment than both MQ and PHC. This observation strongly confirms the benefit of the hybrid form of RBF that can be more reliable. Furthermore, it has to be stated again that the hard work of finding the most suitable shape parameter when 
using MQ still persisted in this case where the values shown here are obtained purely by carrying out a large amount of numerical tests.

Table 4. Comparison of $\%-$ Rel.Err. $\left(\mathbf{x}_{i}\right)$ of solutions numerically obtained by using three RBFs at two different time levels; $t=0.5$ and $t=1.0$; measured at selected locations over the domain.

\begin{tabular}{|c|c|c|c|c|c|c|}
\hline \multirow[b]{2}{*}{$(x, y)$} & \multicolumn{3}{|c|}{$t=0.5$} & \multicolumn{3}{|c|}{$t=1.0$} \\
\hline & $\begin{array}{c}\text { MQ } \\
\left(\varepsilon_{\text {best }}=1.00\right)\end{array}$ & PHC & HyB & $\begin{array}{c}\text { MQ } \\
\left(\varepsilon_{\text {best }}=5.15\right)\end{array}$ & PHC & HyB \\
\hline$(0.6,0.6)$ & $1.61 \mathrm{E}-01$ & $8.62 \mathrm{E}-02$ & $5.24 \mathrm{E}-02$ & $1.971 \mathrm{E}-01$ & $9.84 \mathrm{E}-02$ & $2.77 \mathrm{E}-01$ \\
\hline$(0.8,0.8)$ & $2.35 \mathrm{E}-01$ & $8.05 \mathrm{E}-02$ & $8.10 \mathrm{E}-02$ & $0.774 \mathrm{E}-01$ & $3.02 \mathrm{E}-01$ & $1.59 \mathrm{E}-02$ \\
\hline$(1.0,1.0)$ & $1.47 \mathrm{E}-01$ & 4.44E-01 & $4.17 \mathrm{E}-02$ & $0.690 \mathrm{E}-01$ & $1.02 \mathrm{E}-01$ & $1.25 \mathrm{E}-02$ \\
\hline$(1.2,1.2)$ & $1.94 \mathrm{E}-02$ & $8.14 \mathrm{E}-02$ & $5.05 \mathrm{E}-01$ & $1.704 \mathrm{E}-00$ & $2.51 \mathrm{E}-01$ & $0.94 \mathrm{E}-02$ \\
\hline$(1.4,1.4)$ & $2.04 \mathrm{E}-01$ & $8.56 \mathrm{E}-02$ & 2.79E-02 & $1.204 \mathrm{E}-00$ & $1.10 \mathrm{E}-01$ & $3.04 \mathrm{E}-02$ \\
\hline$(1.6,1.6)$ & $1.44 \mathrm{E}-02$ & $7.12 \mathrm{E}-02$ & $1.61 \mathrm{E}-02$ & $0.821 \mathrm{E}-01$ & $1.57 \mathrm{E}-01$ & $2.02 \mathrm{E}-02$ \\
\hline$(1.8,1.8)$ & $1.29 \mathrm{E}-01$ & $5.50 \mathrm{E}-01$ & $5.82 \mathrm{E}-02$ & $1.604 \mathrm{E}-01$ & $0.91 \mathrm{E}-02$ & $3.30 \mathrm{E}-01$ \\
\hline
\end{tabular}



Fig. 3 Maximum error $\left(L_{\infty}\right)$ measured over the domain at a wide range computational times ( $1.1 \leq t \leq 2.0$ ) with $N^{\chi}=144$.

\section{Conclusion}

In this work, some challenging partial differential equations are numerically solved by the dual reciprocity boundary element method (DRBEM). Unlike the usual manner of applying DRBEM where the main ingredient (the RBF used) depends highly on the choice of its shape parameter, a new form of radial basis function containing no shape is being proposed in this work. Instead, a locally self-autoadaptive weight is introduced to play the main rule. Some numerical benchmarking tests were carried out and some interesting findings are;

1. The new RBF is found to produce results which are slightly better than those provided by MQ or PHC.

2. The difficulty usually encountered when searching for an optimal choice of shape parameter is no longer included, saving both time and effort.

3. For time-dependent problem, the new RBF is seen to preserve good quality of results even when time is further increased where the other two RBF forms are losing their accuracy.

4. A less sensitivity to the number of computational nodes is also observed with using the proposed RBF.

Although some positive and promising aspects have been observed in this work, it is still a challenging task to further investigate under some more complex problems. The dual reciprocity boundary element method (DRBEM) has been widely applied to a branch of engineering problems and as far as 
DRBEM goes, the choice of RBF proposed in this work shall well be an alternative tool in order to get rid of the burden of choosing a good shape parameter. Limitations and challenges, however, might occur when dealing with highly nonlinear PDEs and this remains one of our future research topics.

\section{Acknowledgments}

The corresponding author would like to express his sincere appreciation to the Centre of Excellence in Mathematics, Thailand, for their kind support.

\section{References:}

[1] D. Nardini, and C.A. Brebbia, A new approach to free vibration analysis using boundary elements, Applied Mathematical Modelling, Vol.7, No.3, 1983, pp. 157-162.

[2] K. Chanthawara, S. Kaennakham, and W. Toutip, The Dual Reciprocity Boundary Element Method (DRBEM) with Multiquadric Radial Basis Function for Coupled Burgers' equations, The International Journal of Multiphysics, Vol.8, No.2, 2014, pp. 123-144.

[3] D. Lazzaro, and L.B. Montefusco, Radial basis functions for the multivariate interpolation of large scattered data sets, Journal of Computational and Applied Mathematics, Vol.140, No.1-2, 2002, pp.521-536.

[4] R.L. Hardy, Multiquadric equations of topography and other irregular surfaces, Journal of Geophysical Research, Vol.76, No.8, 1971, pp.1905-1915.

[5] C. Franke, and R. Schaback, Convergence order estimates of meshless collocation methods using radial basis functions, Advances in Computational Mathematics, Vol.8, No.4, 1998, pp.381-399.

[6] X. Zhang, K.Z. Song, M.W. Lu, and X. Liu, Meshless methods based on collocation with radial basis functions, Computational Mechanics, Vol.26, No.4, 2000, pp.333-343

[7] J.G. Wang, and G.R. Liu, On the optimal shape parameters of radial basis functions used for 2-D meshless methods, Computer Methods in Applied Mechanics and Engineering, Vol.191, No.23-24, 2002, pp.2611-2630.

[8] C.K. Lee, X. Liu, and S.C. Fan, Local multiquadric approximation for solving boundary value problems, Computational Mechanics, Vol.30, No.5-6, 2003, pp.396-409.

[9] J. Biazar, and M. Hosami, Selection of an interval for variable shape parameter in approximation by radial basis functions, Advances in Numerical Analysis, Vol.2016, 2016, pp.1-11
[10] S. Kaennakham, and K. Chanthawara, A Numerical Experiment on Optimal Inverse Multiquadric RBF Shape Parameter in the Dual Reciprocity Boundary Element Method for Convective-Dominated Problems, Thai Journal of Mathematics, 2016, pp.119-131.

[11] S. Kaennakham, and N. Chuathong, Solution to a Convection-Diffusion Problem Using a New Variable Inverse-Multiquadric Parameter in a Collocation Meshfree Scheme, International Journal of Multiphysics, Vol.11, No.4, 2017, pp.359-374.

[12] N. Chuathong, and S. Kaennakham, A Numerical Investigation on Variable Shape Parameter Schemes in a Meshfree Method Applied to a Convection-Diffusion Problem, International Journal of Applied Engineering Research, Vol.12, No.14, 2017, pp.4162-4170.

[13] M. Yaghouti, and H.R. Azarboni, Determining optimal value of the shape parameter cc in RBF for unequal distances topographical points by Cross-Validation algorithm, Journal of Mathematical Modeling, Vol.5, No.1, 2017, pp.53-60.

[14] O. Davydov, and D.T. Oanh, On the optimal shape parameter for Gaussian radial basis function finite difference approximation of the Poisson equation, Computers and Mathematics with Applications, Vol.62, No.5, 2011, pp.2143-2161.

[15] M. Ranjbar, A new variable shape parameter strategy for Gaussian radial basis function approximation methods, Annals of the University of Craiova - Mathematics and Computer Science Series,Vol.42, No.2, 2015, pp.260-272.

[16] A. Guessab and A. Aris, Numerical Analysis of CPU with Heat Sink base of Copper Core using CFD, International Journal of Circuits, Systems and Signal Processing, , Volume 14, 2020, pp. 144-148.

[17] M.D.L.D. Vedova and P. Alimhillaj, Novel Fluid Dynamic Nonlinear Numerical Models of Servovalves for Aerospace, Systematic Risk Measurement Based on CoVaR Model, International Journal of Circuits, Systems and Signal Processing, Vol. 14, 2020, pp. 39-51.

[18] E. J. Kansa, Multiquadrics-A scattered data approximation scheme with applications to computational fluid-dynamics-II solutions to parabolic, hyperbolic and elliptic partial differential equations, Computers and Mathematics with Applications, Vol.24, No.12, 1992, pp.99-120.

[19] E.J. Kansa, and R.E. Carlson, Improved accuracy of multiquadric interpolation using variable shape parameters, Computers and 
Mathematics with Applications, Vol.24, No.12, 1992, pp.99-120.

[20] S.A. Sarra, Adaptive radial basis function method for time dependent partial differential equations, Applied Numerical Mathematics, Vol.54, No.1, 2005, pp.79-94.

[21] S.A. Sarra, and D. Sturgill, A random variable shape parameter strategy for radial basis function approximation methods, Engineering Analysis with Boundary Elements, Vol.33, No.11, 2009, pp.1239-1245.

[22] K. Chanthawara, and S. Kaennakham, A localadaptive Multiquadric shape parameter applied with DRBEM to convection-diffusion problem, Journal of Telecommunication, Electronic and Computer Engineering, Vol.11, No.1-9, 2018, pp.141-146.

[23] W. Toutip, The dual reciprocity boundary element method for linear and nonlinear problems, University of Hertfordshire, United Kingdom, 2001.

[24] P.K. Mishra, S.K. Nath, M.K. Sen, and G.E. Fasshauer, Hybrid Gaussian-cubic radial basis functions for scattered data interpolation, Computational Geosciences, Vol.22, No.5, 2018, pp.1203-1218.

[25] S. Kaennakham, and N. Chuathong, An Automatic Node-Adaptive Scheme applied with a RBF-Collocation Meshless Method, Applied Mathematics and Computation, Vol.348, 2019, pp.102-125.

[26] K. Djidjeli, P.P. Chinchapatnam, P.B. Nair and W.G. Price, Global and compact meshless schemes for the unsteady convection-diffusion equation, International symposium on health care and biomedical research interaction, University of Southampton, 2004.

\section{Creative Commons Attribution License 4.0 (Attribution 4.0 International, CC BY 4.0)}

This article is published under the terms of the Creative Commons Attribution License 4.0

https://creativecommons.org/licenses/by/4.0/deed.en_US 